\title{
PROXIMITY MAPS FOR CONVEX SETS
}

\section{WARD CHENEY AND ALLEN A. GOLDSTEIN}

The method of successive approximation is applied to the problem of obtaining points of minimum distance on two convex sets. Specifically, given a closed convex set $K$ in Hilbert space, let $P$ be the map which associates with each point $x$ the point $P x$ of $K$ closest to $x$. That $P$ is well-defined is proved in $[1$, p. 6]. $P$ will be called the proximity map for $K$. If there are two such sets, $K_{1}$ and $K_{2}$, let $Q$ denote the composition $P_{1} P_{2}$ of their proximity maps. It is shown that every fixed point of $Q$ is a point of $K_{1}$ closest to $K_{2}$, and that the fixed points of $Q$ may be obtained by iteration of $Q$ when one of the sets is compact or when both are polytopes in $E_{n}$. An application to the solution of linear inequalities is cited. Our thanks are due the referee for having suggested substantial simplifications.

THEOREM 1. Let $Q$ be a map of a metric space into itself such that

(i) $d(Q x, Q y) \leqq d(x, y)$,

(ii) if $x \neq Q x$, then $d\left(Q x, Q^{2} x\right)<d(x, Q x)$,

(iii) for each $x$, the sequence $Q^{n} x$ has a cluster point. Then for each $x$, the sequence $Q^{n} x$ converges to a fixed point of $Q$.

Proof. By (i), the sequence $d\left(Q^{n} x, Q^{n+1} x\right)$ is nonincreasing. Let $y$ be a cluster point of $Q^{n} x$, say $y=\lim _{k} Q^{n_{k}} x$. By (i), $Q$ is continuous; therefore $d(y, Q y)=\lim _{k} d\left(Q^{n_{k}} x, \quad Q^{n_{k}+1} x\right)=\lim _{n} d\left(Q^{n} x, \quad Q^{n+1} x\right)$ $=\lim _{k} d\left(Q^{n_{k}+1}, Q^{n_{k}+2} x\right)=d\left(Q y, Q^{2} y\right)$ contrary to (ii) unless $y=Q y$. From (i) it follows that for all $n, d\left(Q^{N+n} x, y\right) \leqq d\left(Q^{N} x, y\right)$ whence $Q^{n} x \rightarrow y$.

COROLLARy. Let $Q$ be a map of a normed linear space into itself having the property $\|Q x-Q y\| \leqq\|x-y\|$, equality holding only if $x=y$. Let $R=\alpha Q+(1-\alpha) I,(0<\alpha \leqq 1)$. If the range of $R$ is compact, then $Q$ has a unique fixed point which is the limit of every sequence $R^{n} x$ with $x$ arbitrary. (For related results, see [2].)

Lemma. Let $K$ be a convex set in Hilbert space. A point $b \in K$ is nearest $a$ point $a \notin K$ if and only if $s \equiv(x-b, b-a) \geqq 0$ for all $x \in K$.

Proof. Suppose $b$ nearest $a$, and let $x$ be arbitrary in $K$. When $0 \leqq t \leqq 1, t x+(1-t) b \in K$. Thus $0 \leqq\|a-t x-(1-t) b\|^{2}-\|a-b\|^{2}$ $=t^{2}\|b-x\|^{2}+2 t s$. But this inequality would be violated by small $t$

Presented to the Society, January 22, 1959; received by the editors July 26, 1958 and, in revised form, October 4, 1958. 
unless $s \geqq 0$. For the converse, suppose $s \geqq 0$. Then $\|x-a\|^{2}-\|a-b\|^{2}$ $=(x, x)-2(a, x)+2(a, b)-(b, b)=(x-b, x-b)+2 s \geqq 0$.

Theorem 2. Let $K_{1}$ and $K_{2}$ be two closed convex sets in Hilbert space. Let $P_{i}$ denote the proximity map for $K_{i}$. Any fixed point of $P_{1} P_{2}$ is a point of $K_{1}$ nearest $K_{2}$, and conversely.

Proof. Suppose $y=P_{2} x$ and $x=P_{1} y$. If $x=y$, the distance between $K_{1}$ and $K_{2}$ is thereby attained. Otherwise $x \notin K_{2}$ and $y \notin K_{1}$. If $u$ is arbitrary in $K_{1}$, then by the Lemma, $(u-x, x-y) \geqq 0$ whence $(u, x-y)$ $\geqq(x, x-y)$. Similarly for arbitrary $v \in K_{2},(v, y-x) \geqq(y, y-x)$. Addition yields $(u-v, x-y) \geqq(x-y, x-y)$ from which via the Schwartz inequality, $\|u-v\| \geqq\|x-y\|$. For the converse, suppose that $\| x-P_{2} x$ $\leqq\left\|z-P_{2} z\right\|$ for all $z \in K_{1}$. Setting $z=P_{1} P_{2} x$ we have $\left\|z-P_{2} z\right\|$ $\leqq\left\|z-P_{2} x\right\| \leqq\left\|x-P_{2} x\right\| \leqq\left\|z-P_{2} z\right\|$ whence $z=x$ by the uniqueness of $z$.

Theorem 3. The proximity map $P$ for a closed convex set $K$ in Hilbert space satisfies the Lipschitz condition $\|P x-P y\| \leqq\|x-y\|$, equality holding only if $\|x-P x\|=\|y-P y\|$.

Proof. By the Lemma, $A \equiv(P x-P y, P y-y) \geqq 0$ and $B \equiv(P y-P x$, $P x-x) \geqq 0$. Regrouping terms in the inequality $A+B \geqq 0$ and using the Schwartz inequality, one has $\|P y-P x\| \cdot\|y-x\| \geqq(P y-P x$, $y-x) \geqq(P y-P x, P y-P x)=\|P y-P x\|^{2}$, whence $\|y-x\| \geqq\|P y-P x\|$. Equality holds here only if $A=B=0$ and if $P y-P x=\lambda(y-x)$. Thus $C \equiv(y-x, P y-y)=0$ and $D \equiv(y-x, P x-x)=0$. A computation shows then that $0=A-B+C+D=\|P x-x\|^{2}-\|P y-y\|^{2}$.

Theorem 4. Let $K_{1}$ and $K_{2}$ be two closed convex sets in Hilbert space and $Q$ the composition $P_{1} P_{2}$ of their proximity maps. Convergence of $Q^{n} x$ to a fixed point of $Q$ is assured when either (a) one set is compact, or (b) one set is finite dimensional and the distance between the sets is attained.

Proof. Theorem 3 implies that $Q$ satisfies (i) of Theorem 1. If $y \equiv Q x \neq x \in K_{1}$, then $\left\|y-P_{2} y\right\| \leqq\left\|y-P_{2} x\right\|<\left\|x-P_{2} x\right\|$. By Theorem 3, $\|Q x-Q y\| \leqq\left\|P_{2} x-P_{2} y\right\|<\|x-y\|$. Thus $Q$ satisfies (ii) of Theorem 1. If the distance between the sets is attained, then $Q$ has a fixed point $y$ by Theorem 2 , and $\left\|Q^{n} x\right\| \leqq\left\|Q^{n} x-Q y\right\|+\|y\| \leqq\|x-y\|+\|y\|$. Boundedness of $\left\|Q^{n} x\right\|$ and finite-dimensionality of $K_{1}$ suffice for (iii) of Theorem 1. $Q^{\prime}=P_{2} P_{1}$ replaces $Q$ in these arguments when $K_{2}$ replaces $K_{1}$. But if $y$ is a fixed point of $Q^{\prime}$, then $P_{1} y$ is a fixed point of $Q$.

THEOREM 5. In a finite dimensional Euclidean space, the distance 
between two polytopes is attained, a polytope being the intersection of a finite family of halfspaces.

Proof. First, the case when both polytopes are linear manifolds. Let $K_{1}$ be the linear span of $\left\{x_{1}, \cdots, x_{m}\right\}$ and $K_{2}$ the linear span of $\left\{y_{1}, \cdots, y_{n}\right\}$ translated by a vector $c y_{0}$. Assume the $x$ 's and $y$ 's each form orthonormal sets. A point $x=\sum \xi_{i} x_{i}$ is sought which minimizes $G=\left\|c y_{0}+\sum\left(x, y_{i}\right) y_{i}-x\right\|^{2} . G$ is a positive definite quadratic function of $\xi_{1}, \cdots, \xi_{m}$, and therefore attains a minimum.

Now assume the validity of the theorem when one polytope is of dimension less than $n$ and the other is a linear manifold. (The validity for $n=1$ being established by the above.) Let $K_{1}$ be a polytope of dimension $n$ and $K_{2}$ a linear manifold. On each proper face of $K_{1}$ there is a point nearest $K_{2}$. There being only a finite number of faces, either one of these points is the required one or there is a point $x_{0} \in K_{1}$ such that $d\left(x_{0}, K_{2}\right)<d\left(F, K_{2}\right)$ for all proper faces $F$ of $K_{1}$. In the latter case, let $y_{0}$ be chosen nearest to $K_{2}$ in the least linear manifold containing $K_{1}$. Since $d\left(x, K_{2}\right)$ is a convex function of $x$, the line segment $x_{0} y_{0}$ contains no point of any proper face of $K_{1}$. Therefore $y_{0} \in K_{1}$ and must be the required point. The proof for the remaining case is as above, mutatis mutandis.

Linear inequalities. Consider the system of linear inequalities $\left(A^{i}, x\right) \leqq b_{i}$ where $x \in E_{n}$ and $1 \leqq i \leqq m$. Let $K_{1}$ denote the range of the matrix $A$ and $K_{2}$ the orthant $\left\{y \in E_{m}: y_{i} \leqq b_{i}\right.$ all $\left.i\right\}$. If $K_{1}$ and $K_{2}$ have a point in common, then any $x$ for which $A x \in K_{1} \cap K_{2}$ is a solution of the system. Even if the system is inconsistent, a point on $K_{1}$ closest to $K_{2}$ may be obtained by iteration of the map $Q=P_{1} P_{2}$. These proximity maps are defined as follows. If $A$ is of rank $n$, $P_{1}=A\left(A^{T} A\right)^{-1} A^{T}$. If $A$ is not necessarily of rank $n$, one may write $P_{1}=B B^{T}$ where $B$ is a column-orthogonal matrix whose range is that of $A . P_{2} y=z$ if and only if $z_{i}=y_{i}$ when $y_{i} \leqq b_{i}$ and $z_{i}=b_{i}$ otherwise. Convergence of each sequence $Q^{n} x$ is guaranteed by Theorem 4 .

\section{BIBLIOGRAPHY}

1. B. v.-Sz. Nagy, Spektraldarstellung Linearer Transformationen des Hilbertschen Raumes, Berlin, 1942.

2. H. Schaefer, Über die Methode Sukzessiver Approximationen, Jber. Deutsch. Math. Verein. vol. 59 (1957) pp. 131-140.

Convair Astronautics, San Diego 